\title{
De novo degenerative lumbar scoliosis: a systematic review of prognostic factors for curve progression
}

\author{
Sayf S. A. Faraj ${ }^{1}$ Roderick M. Holewijn ${ }^{1} \cdot$ Miranda L. van Hooff ${ }^{2} \cdot$ \\ Marinus de Kleuver ${ }^{1,2} \cdot$ Ferran Pellisé $^{3} \cdot$ Tsjitske M. Haanstra $^{1}$
}

Received: 31 December 2015/Revised: 29 March 2016/Accepted: 15 May 2016/Published online: 24 May 2016

(C) The Author(s) 2016. This article is published with open access at Springerlink.com

\begin{abstract}
Purpose To identify prognostic factors for curve progression in de novo degenerative lumbar scoliosis (DNDLS) by performing a systematic review of the literature.

Methods Studies were selected for inclusion following a systematic search in the bibliographic databases PubMed and EMBASE prior to September 2015 and hand searches of the reference lists of retrieved articles. Two authors independently assessed methodological quality. Data were extracted and presented according to a best evidence synthesis.

Results The literature search generated a total of 2696 references. After removing duplicates and articles that did not meet inclusion criteria, 12 studies were included. Due to the lack of statistical analyses, pooling of data was not possible. Strong evidence indicates that increasing intervertebral disk degeneration, lateral vertebral translation $\geq 6 \mathrm{~mm}$, and an intercrest line through L5 (rather than L4) are associated with DNDLS curve progression. Moderate evidence suggests that apical vertebral rotation Grade II or III is associated with curve progression. For the majority of other prognostic factors, we found limited, conflicting, or
\end{abstract}

Electronic supplementary material The online version of this article (doi:10.1007/s00586-016-4619-9) contains supplementary material, which is available to authorized users.

Tsjitske M. Haanstra

t.haanstra@vumc.nl

1 Department of Orthopaedic Surgery, VU University Medical Center, De Boelelaan 1117, 1081 HV Amsterdam, The Netherlands

2 Department of Orthopaedic Surgery, Sint Maartenskliniek, Nijmegen, The Netherlands

3 Spine Unit, Hospital Vall d'Hebron, Barcelona, Spain inconclusive evidence. Osteoporosis, a coronal Cobb angle $<30^{\circ}$, lumbar lordosis, lateral osteophytes difference of $\geq 5 \mathrm{~mm}$, and degenerative spondylolisthesis have not been shown to be risk factors. Clinical risk factors for progression were not identified.

Conclusions This review shows strong evidence that increased intervertebral disk degeneration, an intercrest line through L5, and apical lateral vertebral translation $\geq 6 \mathrm{~mm}$ are associated with DNDLS curve progression. Moderate evidence was found for apical vertebral rotation (Grade II/III) as a risk factor for curve progression. These results, however, may not be directly applicable to the individual patient.

Keywords De novo degenerative lumbar scoliosis . Adult's scoliosis - Prognostic factors · Curve progression . Systematic review

\section{Introduction}

The Global Burden of Disease Study has shown that low back pain has remained the leading cause for years lived with disability (YLD) in Western societies in the last two decades [1]. With its lifetime prevalence between 58 and $84 \%$, low back pain poses substantial burden on global health care [2-4]. A large proportion (85\%) of low back pain is non-specific and has an unknown aetiology [5]. Of the remaining group, one of the known causes for low back pain is scoliosis: a lateral and rotational deformity of the spine with a Cobb angle of more than $10^{\circ}$ in the coronal plain $[6,7]$. Primary degenerative scoliosis or de novo degenerative lumbar scoliosis (DNDLS) is a condition in which a lumbar scoliotic curve typically develops after the age of 50 in patients who did not have a childhood scoliosis 
[7, 8]. DNDLS causes an increasing burden on society in aging populations, with reduction in health related quality of life due to severe back and leg pain $[9,10]$. The aetiology of DNDLS is multifactorial, including genetic predisposition and intervertebral disk degeneration [11-15]. Several studies have reported prevalence rates of 8.3, 8.9, and $13.3 \%$ for adult scoliosis [16-18]. There is sufficient evidence to assume that the growing elderly population will result in an increase in prevalence rates of adult scoliosis [19-22].

DNDLS can cause back and leg pain symptoms in patients, without a history of adolescent idiopathic scoliosis (AIS), and is radiologically characterized by a Cobb angle of $10^{\circ}$ or more, and by asymmetrical disk and/or facet arthritis [7, 23-27]. In DNDLS, to date, curve progression is unpredictable. Weinstein and Ponseti 1983 showed that $68 \%$ of curves in AIS as well as adult scoliosis progressed more than $5^{\circ}$ after skeletal maturity [28]. In addition, they demonstrated that the progression rate in adult scoliosis is much higher in comparison with AIS. Little else is known about curve progression in DNDLS, and prognostic factors are still undefined. Estimating the risk of curve progression is essential for health care providers to be able to adequately inform patients, and to determine the optimal timing for therapeutic interventions.

The aim of this study was to identify clinical and radiological prognostic factors for curve progression through a systemic review of the literature.

\section{Methods}

\section{Search strategy}

The review protocol was developed with the use of the Preferred Reporting Items for Systematic Reviews and Meta-Analyses (PRISMA) and MOOSE guidelines [29, 30]. Relevant published studies involving prognostic factors of progression in DNDLS that were published prior to September 2015 were identified by a systematic search of Pubmed and Embase conducted by an experienced medical information specialist, and backwards citation of obtained articles. Search terms expressing 'degenerative scoliosis' and 'adult scoliosis' were used in combination with search terms comprising 'progression'. Keywords used to identify a relevant design were: longitudinal studies, observational studies, prospective studies, cohort studies, retrospective studies, or follow-up studies in the title or in the abstract (Table 1; Supplementary Material). Two reviewers (SSAF and $\mathrm{RMH}$ ) independently screened the titles, abstracts, and full text articles identified by the literature search. Full text articles were retrieved if the abstract passed the first eligibility screening or provided insufficient information.

\section{Selection criteria}

The following inclusion criteria were applied: (1) de novo degenerative lumbar scoliosis, (2) adult scoliosis, (3) curve progression determined by increase in Cobb angle, (4) study population of $N \geq 10$, (5) studies published in the English language, and (6) observational studies. Exclusion criteria were: (1) animal study, (2) biomechanical studies, (3) prior juvenile, adolescent, associated congenital, developmental, or neuromuscular spinal abnormalities, or (4) spinal surgery. All obtained full text articles were reviewed for inclusion independently by SSAF and RMH.

\section{Data extraction}

A data-extraction form was developed to obtain the following information: authors, year of publication, definition of DNDLS and curve progression, initial and final Cobb angle, follow-up years, number of patients, gender, researched prognostic factors, including their measurement methods, and statistical analyses method(s).

\section{Methodological quality}

Selected papers were subjected to a quality assessment based on the criteria developed by Hayden et al. to assess the methodological quality of studies on prognostic factors [31]. The following domains were evaluated: (1) study participation, (2) study attrition, (3) measurement of prognostic factors (4) adjustment for confounding, (5) measurement of outcomes, and (6) analysis approach. For this study, the aim is not to explore the causality of the association between a specific factor and the outcome, but to estimate the probability of an outcome. Therefore, the Hayden criteria were modified (excluding confounding), as shown in Table 2 (Supplementary Material). These items were scored with 1 point if the item was sufficiently fulfilled. In the case of missing data or insufficient information, 0 points were given. A total quality score was calculated for each study by summing the fulfilled items. High quality was defined as a quality score of $\geq 9$ and low quality $<9$. The same approach has also been applied in studies on the prognostic factors of knee and hip arthritis [32, 33]. All included articles were scored independently by SSAF and RMH. In the case of disagreement, the authors arranged a consensus-meeting. When disagreement persisted, a third independent reviewer (TMH) made the final decision.

\section{Analysis}

Correlation coefficients were statistically pooled if there was sufficient clinical and statistical homogeneity 
regarding the definition of DNDLS and curve progression, study population, measured determinants, and assessed outcomes. In the absence of statistical analysis (correlation or beta coefficients) and heterogeneity in measurement methods of outcomes, region, sample size, and study design, the strength of evidence for prognostic factors was assessed according to the best evidence synthesis (Table 3; Supplementary Material). Level of evidence was based on the updated guidelines by Furlan et al. [34]. Prognostic factors were recorded as consistent if more than $75 \%$ of the studies reported the same direction of association. In reporting the results, the prognostic factors were grouped into patient and radiographic characteristics.

\section{Results}

\section{Studies included}

The literature search generated a total of 2696 references: 1323 articles in PubMed and 1373 in EMBASE. After removing duplicates of references, 1696 papers remained. Of these 1696 papers, we identified 10 papers that met our inclusion criteria. In addition, two studies were found eligible by backward citation. In total, 12 studies were included (Fig. 1).

\section{Methodological quality}

The overall interobserver agreement was 0.88; kappa $=0.74$, representing good agreement [35]. Of the 12 included studies, 6 were of high quality ( $\geq 9$ points $[13,36-$ 40]). Most methodological shortcomings concerned lack of adequate follow-up (item $\mathrm{F}$ and $\mathrm{G}$ ), blinding (item $\mathrm{H}$ and J), and statistical analysis (item L and M) (Table 4; Supplementary Material).

\section{Study characteristics (Table 1)}

The sample size varied between 14 and 318 patients, with a total of 985 . Three studies included over 100 patients [6, $18,41]$. The shortest follow-up was 2 years, and the longest was 30 years. In two studies, the follow-up period was not available $[11,18]$. A variety in definitions for DNDLS and curve progression was found.

\section{Study results (Tables 2 and 3)}

A total of 31 prognostic factors were found, and each of these was classified as either patient or radiographic characteristic. A full overview of the prognostic factors, their measurement methods, and their association with DNDLS curve progression is presented in Tables 2 and 3. Most authors did not report or perform a correlation analysis of the determinant's association with DNDLS curve progression. Due to a lack of sufficient studies with statistical data, pooling was not possible. In addition, a large heterogeneity between studies in measurement methods, sample sizes, regional differences, and variations in DNDLS and curve progression definitions also precluded statistical pooling of the results. Therefore, it was necessary to summarize each determinant according to the best evidence synthesis to determine the strength of the association with curve progression.

\section{Best evidence synthesis (Fig. 2)}

The amount of intervertebral disk degeneration (the ratio of disk height on one decreased side divided by the disk height on the other side) and a lateral vertebral translation of more than $\geq 6 \mathrm{~mm}$ were both found to be strongly associated with inducing DNDLS curve progression. Patients with the intercrest line passing through the L5 vertebra had a larger final scoliosis angle than patients with the intercrest line passing through the L4 vertebra $[8,18$, $38,40,41]$.

Moderate evidence suggests that more apical vertebral rotation ( $>$ Grade II or III Nash and Moe's method [42]) is associated with DNDLS curve progression.

Strong evidence suggests that there is no association between curve progression and osteoporosis, which was measured with several methods (X-ray, biopsy, and DEXA). Other findings with strong evidence for no association were a Cobb angle $<30^{\circ}$, lumbar lordosis, and lateral osteophytes difference $\geq 5 \mathrm{~mm}$ (measured as the difference between the length of the lateral osteophytes on each side on an AP radiograph).

Moderate evidence was found that there was no association between DNDLS curve progression and age, gender, an intercrest line through the L4 vertebra, apical lateral vertebral translation $<6 \mathrm{~mm}$, and the side of convexity of the scoliosis. Limited evidence was found between degenerative spondylolisthesis and curve progression. For other prognostic factors, limited, inconclusive, or inconsistent evidence was found.

\section{Discussion}

The aim of this study was to identify prognostic factors associated with DNDLS curve progression through a systematic review of the literature. After an extensive systematic search of the literature, we identified 12 papers of varying methodological quality. Despite inherent design weaknesses in most papers (mostly related to the difficulty of studying the natural history of a disease), using a 
Fig. 1 PRISMA flow chart of the study search and selection [29]

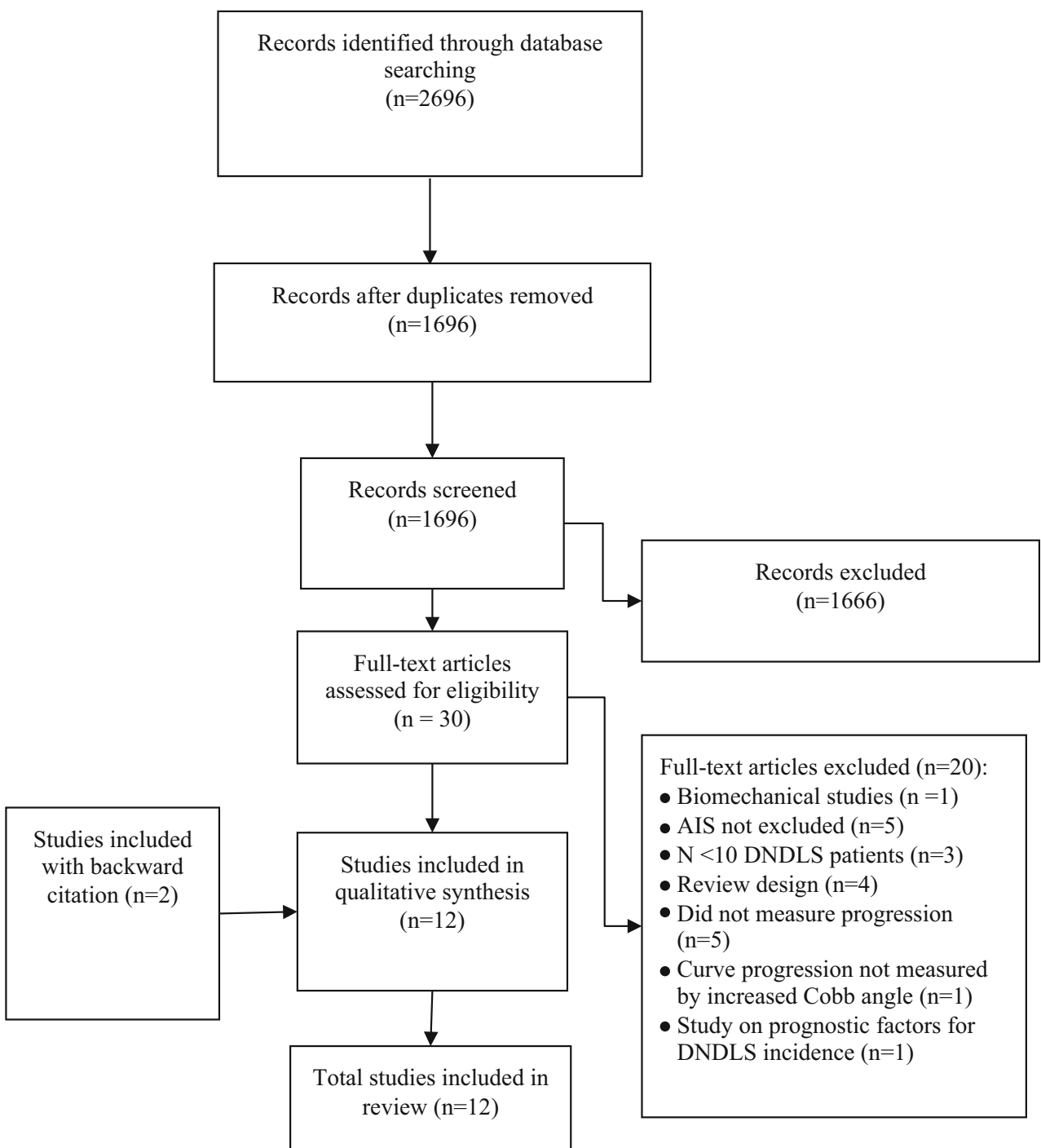

Table 1 Overview of study characteristics

\begin{tabular}{|c|c|c|c|c|c|c|c|}
\hline References & $\begin{array}{l}\text { Definition } \\
\text { DNDLS }\end{array}$ & $\begin{array}{l}\text { Definition curve } \\
\text { progress }\end{array}$ & $\begin{array}{l}\text { No. patients } \\
\text { (women) }\end{array}$ & $\begin{array}{l}\text { Age, } \\
\text { years }\end{array}$ & $\begin{array}{l}\text { Initial Cobb } \\
\text { angle }\end{array}$ & $\begin{array}{l}\text { Final Cobb } \\
\text { angle }\end{array}$ & $\begin{array}{l}\text { Follow-up, } \\
\text { years }\end{array}$ \\
\hline Vanderpool et al. [11] & $\geq 7^{\circ}$ & $>0^{\circ}$ & $14(11)$ & 67 & $\mathrm{n} / \mathrm{a}$ & $7^{\circ}-53^{\circ}$ & $\mathrm{n} / \mathrm{a}$ \\
\hline Robin et al. [6] & $\geq 10^{\circ}$ & $\geq 5^{\circ}$ & 179 (133) & $45-84^{\mathrm{a}}$ & $0^{\circ}-20^{\circ}$ & $\mathrm{n} / \mathrm{a}$ & $7-13$ \\
\hline Pritchett et al. [8] & $\geq 10^{\circ}$ & $\geq 10^{\circ}$ & $41(\mathrm{n} / \mathrm{a})$ & $69(50-89)$ & $\geq 10^{\circ}$ & $14^{\circ}-60^{\circ}$ & $12(10-30)$ \\
\hline Korovessis et al. [36] & $\geq 10^{\circ}$ & $\geq 5^{\circ}$ & $91(73)$ & $67 \pm 9$ & $16.5^{\circ}$ & $\mathrm{n} / \mathrm{a}$ & $3.7(2-5)$ \\
\hline Sapkas et al. [41] & $\geq 10^{\circ}$ & $\geq 10^{\circ}$ & $162(162)$ & $65(45-84)$ & $\geq 10^{\circ}$ & $12^{\circ}-50^{\circ}$ & $\geq 10$ \\
\hline Murata et al. [37] & $\geq 10^{\circ}$ & $\geq 3^{\circ}$ & $47(33)$ & $40-75^{\mathrm{a}}$ & $11.6^{\circ}(10-26.5)$ & $\mathrm{n} / \mathrm{a}$ & $10(7-14)$ \\
\hline Kobayashi et al. [13] & $\geq 10^{\circ}$ & $\geq 5^{\circ}$ & $22(\mathrm{n} / \mathrm{a})$ & $72(60-95)$ & $\mathrm{n} / \mathrm{a}$ & $10-18$ & $12(10-15)$ \\
\hline Chin et al. [38] & $\geq 3^{\circ}$ & $>0^{\circ}$ & $24(17)$ & $68(50-81)$ & $14.2^{\circ}\left(3^{\circ}-30^{\circ}\right)$ & $21.6^{\circ}\left(4^{\circ}-46^{\circ}\right)$ & 5 \\
\hline Kohno et al. [39] & $\geq 5^{\circ}$ & $>0^{\circ}$ & $27(20)$ & $63(48-83)$ & $10.1^{\circ}\left(5^{\circ}-20^{\circ}\right)$ & $15.4^{\circ}\left(2^{\circ}-39^{\circ}\right)$ & $12(10-18)$ \\
\hline Seo et al. [40] & $\geq 10^{\circ}$ & $>0^{\circ}$ & $27(24)$ & $64(50-71)$ & $\geq 10^{\circ}$ & $25^{\circ}(\mathrm{SD}, 5.4)$ & 10 \\
\hline Watanuki et al. [43] & $\geq 10^{\circ}$ & $\geq 5^{\circ}$ & $33(23)$ & $75(57-90)$ & $\mathrm{n} / \mathrm{a}$ & $10^{\circ}-26^{\circ}$ & 15 \\
\hline $\mathrm{Xu}$ et al. [18] & $\geq 10^{\circ}$ & $\geq 5^{\circ}$ & $318(265)$ & $72 \pm 10$ & $\mathrm{n} / \mathrm{a}$ & $10^{\circ}-40^{\circ}$ & $\mathrm{n} / \mathrm{a}$ \\
\hline
\end{tabular}

n/a, not available

a Age at initial examination 
Table 2 Reported patient characteristics as prognostic factor for DNDLS curve progression

\begin{tabular}{|c|c|c|c|c|c|}
\hline Determinants ( $N$ patients) & References & Study quality & $\begin{array}{l}\text { Measurement } \\
\text { methods }\end{array}$ & $\begin{array}{l}\text { Statistical } \\
\text { analysis }\end{array}$ & $\begin{array}{l}\text { Association with } \\
\text { curve progression }\end{array}$ \\
\hline \multirow[t]{5}{*}{ Patient age $(N=482)$} & Korovessis et al. [36] & High & Continuous (years) & Not provided & o \\
\hline & Kobayashi et al. [13] & High & Continuous (years) & Not provided & o \\
\hline & Chin et al. [38] & High & Dichotomous & Not provided & + \\
\hline & Seo et al. [40] & High & $<60$ vs $60-70$ vs $\geq 70$ years & $\rho: 0.14$ & o \\
\hline & $\mathrm{Xu}$ et al. [18] & Low & $<65$ vs $\geq 65$ years & $r=0.021 p>0.12$ & o \\
\hline \multirow[t]{5}{*}{ Female $(N=482)$} & Korovessis et al. [36] & High & & Not provided & o \\
\hline & Kobayashi et al. [13] & High & & Not provided & o \\
\hline & Chin et al. [38] & High & & Not provided & + \\
\hline & Seo et al. [40] & High & & Not provided & $\mathrm{o}$ \\
\hline & $\mathrm{Xu}$ et al. [18] & Low & & Not provided & o \\
\hline \multirow[t]{2}{*}{ BMI $(N=340)$} & Kobayashi et al. [13] & High & $\mathrm{kg} / \mathrm{m}^{2}$ & Not provided & o \\
\hline & $\mathrm{Xu}$ et al. [18] & Low & & $r=0.015$ & o \\
\hline \multirow{4}{*}{$\begin{array}{l}\text { Back pain and/or } \\
\text { disability }(N=367)\end{array}$} & Kobayashi et al. [13] & High & JOA & Not provided & $\mathrm{o}$ \\
\hline & Seo et al. [40] & High & ODI & $\rho: 0.6, p<0.05$ & + \\
\hline & Seo et al. [40] & High & VAS & $\rho: 0.16, p>0.05$ & o \\
\hline & $\mathrm{Xu}$ et al. [18] & Low & VAS & $r=0.253, p>0.05$ & o \\
\hline Follow-up period $(N=27)$ & Kohno et al. [39] & High & & $r=0.006, p>0.05$ & o \\
\hline
\end{tabular}

$\mathrm{o}$, no correlation/no relationship found between prognostic factor and curve progression; + , positive association between prognostic factor and increased curve progression; JOA, Japanese Orthopedic Association Score; ODI, Oswestry Disability Index; VAS, Visual Analog Scale; $p$, $p$ value; $r$, correlation coefficient (Pearson's); $\rho$, correlation coefficient (Spearman's)

qualitative best evidence synthesis, we identified several prognostic factors, all of which were radiological.

In light of the continuously expanding global societal problem of low back pain and degenerative scoliosis related to the aging population, it is important to identify the risks of curve progression to be able to adequately inform our patients about their prognosis, and to streamline the crucial process of shared decision making when identifying the right patients for treatment.

\section{Strong evidence}

This review shows strong evidence that the following radiological variables are associated with DNDLS curve progression (Fig. 2):

- Increased intervertebral disk degeneration;

- An intercrest line through L5 (rather than L4 or higher);

- Apical lateral vertebral translation $\geq 6 \mathrm{~mm}$.

This study found that increasing intervertebral disk degeneration (IDD) measured by disk index (ratio of disk height on the decreased side to the disk height on the opposite side) is strongly associated with DNDLS curve progression (Fig. 2). IDD has also been reported to induce DNDLS. Kobayashi et al. found an increased incidence of DNDLS when there was more than $20 \%$ decrease in asymmetrical unilateral disk height due to degenerative processes [13]. Interestingly, only one paper (Watanuki et al.) with a small sample size and limited follow-up rate did not identify IDD to be a significant risk factor for curve progression in DNDLS [43].

Based on Pritchett et al. [8], Chin et al. [38], and Sapkas et al. [41], an intercrest line through the L5 vertebra is a strong prognostic factor for curve progression. On the other hand, no curve progression occurred when the intercrest line went through the L4 vertebra. This seems to indicate that in case of a "deep-seated L5" vertebra, and therefore, a deep seated L4-5 disk, curve progression is less likely to occur $[8,38,40,41]$. A possible explanation for this might be that the less constrained L4-5 disk and L4 vertebra allows L4 to shift and tilt at the lower end of the curve toward the convexity of the curve possibly triggering curve progression $[8,40]$. However, it could be possible that on a PA view the coronal projection of L5 would move upward in case of pelvic retroversion. As such, there could be a correlation between a high L5 and pelvic retroversion and sagittal unbalance. To the best of our knowledge, no prior studies have been conducted on the potential correlation between the intercrest line and pelvic retroversion. All studies included in this review did report a consistent method of standing plain radiographs making that it is less likely that these findings could be attributed to an inconsistent method or positioning of the patient during radiographic examination (Table 3). Further research would add 
Table 3 Reported radiographic characteristics as prognostic factor for DNDLS curve progression

\begin{tabular}{|c|c|c|c|c|c|}
\hline Determinant ( $N$ patients) & References & $\begin{array}{l}\text { Study } \\
\text { quality }\end{array}$ & Measurement methods & $\begin{array}{l}\text { Statistical } \\
\text { analysis }\end{array}$ & $\begin{array}{l}\text { Association } \\
\text { with curve } \\
\text { progression }\end{array}$ \\
\hline \multirow[t]{3}{*}{$\begin{array}{l}\text { Intervertebral disk degeneration } \\
\quad \text { (IDD) }(N=151)\end{array}$} & Korovessis et al. [36] & High & $\begin{array}{l}\text { Ratio of disk height on the decreased side to } \\
\text { the disk height on the opposite side }\end{array}$ & $r=0.75-0.8$ & + \\
\hline & Seo et al. [40] & High & $\begin{array}{l}\text { Ratio of disk height on one (decreased) side } \\
\text { divided by the disk height on the other side } \\
\text { (disk index L3; apex) }\end{array}$ & $\rho: 0.7$ & + \\
\hline & Watanuki et al. [43] & Low & $\begin{array}{l}\text { Intervertebral disk angle: between the } \\
\text { tangential lines of the inferior endplate of a } \\
\text { vertebra and the superior endplate of the } \\
\text { next }\end{array}$ & $p<0.05$ & o \\
\hline \multirow[t]{4}{*}{ Wedging $(N=269)$} & Sapkas et al. [41] & Low & Cobb's lateral vertebral wedging method & Not provided & o \\
\hline & Murata et al. [37] & High & $\begin{array}{l}\text { Angle between the line of a vertebra and the } \\
\text { line of the immediate caudal vertebra } \\
\text { (scoliotic wedging) }\end{array}$ & Not provided & + \\
\hline & Seo et al. [40] & High & $\begin{array}{l}\text { The sum of segmental wedging angles above } \\
\text { and below L3 }\end{array}$ & $\rho: 0.6$ & + \\
\hline & Watanuki et al. [43] & Low & Tilting angle L1-5 & $p<0.05$ & o \\
\hline Vertebral index $(N=22)$ & Kobayashi et al. [13] & High & $\begin{array}{l}\text { The ratio of vertebral height on one } \\
\text { (decreased) side divided by vertebral } \\
\text { height on the other side at L4 vertebrae }\end{array}$ & Not provided & o \\
\hline Bone atrophy $(N=22)$ & Kobayashi et al. [13] & High & Grade $0-3^{\mathrm{C}}$ & Not provided & o \\
\hline \multirow[t]{6}{*}{ Osteoporosis $(N=727)$} & Vanderpool et al. [11] & Low & Bone refraction and biopsy & Not provided & + \\
\hline & Robin et al. [6] & Low & Bone refraction & Not provided & o \\
\hline & Sapkas et al. [41] & Low & DPA $^{153}$ GD to measure BMD & Not provided & o \\
\hline & Kohno et al. [39] & High & Jikei method (based upon X-ray picture) & $p>0.05$ & o \\
\hline & Seo et al. [40] & High & DEXA & $p>0.05$ & o \\
\hline & $\mathrm{Xu}$ et al. $[18]$ & Low & DEXA & $r=0.028$ & o \\
\hline \multirow[t]{5}{*}{ Direction of scoliosis $(N=268)$} & Vanderpool et al. [11] & Low & Left vs right convex & Not provided & o \\
\hline & Pritchett et al. [8] & Low & Left vs right convex & Not provided & o \\
\hline & Sapkas et al. [41] & Low & Left vs right convex & Not provided & o \\
\hline & Seo et al. [40] & High & Left vs right convex & $p>0.05$ & o \\
\hline & Chin et al. [38] & High & Left vs right convex & $p<0.05$ & + \\
\hline $\begin{array}{l}\text { Lateral osteophytes diff. }>5 \mathrm{~mm} \\
\quad(N=27)\end{array}$ & Seo et al. [40] & High & $\begin{array}{l}\text { The substraction of length of lateral } \\
\text { osteophytes on each side }^{\mathrm{B}}\end{array}$ & $p>0.05$ & o \\
\hline \multirow[t]{3}{*}{ Sagittal balance $(N=82)$} & Kobayashi et al. [13] & High & Increase in $\mathrm{C} 7$ plumbline & Not provided & o \\
\hline & Watanuki et al. [43] & Low & & $p>0.05$ & o \\
\hline & Seo et al. [40] & High & & $\rho: 0.6$ & + \\
\hline $\begin{array}{l}\text { Decreased sacral inclination } \\
(N=91)\end{array}$ & Korovessis et al. [36] & High & $\begin{array}{l}\text { Angle formed by the upper end plate of S1 } \\
\text { and the horizontal line }\end{array}$ & $\begin{array}{l}r=0.97- \\
0.99\end{array}$ & o \\
\hline \multirow[t]{2}{*}{ Pelvic tilt $(N=351)$} & Watanuki et al. [43] & Low & Pelvic tilt & $p>0.05$ & o \\
\hline & $\mathrm{Xu}$ et al. [18] & Low & & $p>0.05$ & o \\
\hline Thoracic kyphosis $(N=340)$ & $\begin{array}{l}\text { Kobayashi et al. [13] } \\
\text { Xu et al. [18] }\end{array}$ & $\begin{array}{l}\text { High } \\
\text { Low }\end{array}$ & $\begin{array}{l}\text { From the upper endplate of } \mathrm{T} 4 \text { to lower } \\
\text { endplate of } \mathrm{T} 12\end{array}$ & $\begin{array}{l}\text { Not provided } \\
p>0.05\end{array}$ & $\begin{array}{l}\mathrm{o} \\
\mathrm{o}\end{array}$ \\
\hline \multirow[t]{7}{*}{ Lumbar lordosis $(N=562)$} & Korovessis et al. [36] & High & Distance from the upper end plate of L1 to & $r=0.89-0.98$ & o \\
\hline & Kobayashi et al. [13] & High & lower end plate of L5 & Not provided & o \\
\hline & Murata et al. [37] & High & & Not provided & + \\
\hline & Seo et al. [40] & High & & $p>0.05$ & o \\
\hline & Chin et al. [38] & High & & Not provided & o \\
\hline & Watanuki et al. [43] & Low & & $p>0.05$ & o \\
\hline & Xu et al. [18] & Low & & $p>0.05$ & $\mathrm{o}$ \\
\hline
\end{tabular}


Table 3 continued

\begin{tabular}{|c|c|c|c|c|c|}
\hline Determinant ( $N$ patients) & References & $\begin{array}{l}\text { Study } \\
\text { quality }\end{array}$ & Measurement methods & $\begin{array}{l}\text { Statistical } \\
\text { analysis }\end{array}$ & $\begin{array}{l}\text { Association } \\
\text { with curve } \\
\text { progression }\end{array}$ \\
\hline $\begin{array}{l}\text { Sec. compensatory curve at L4- } \\
\text { S1 }\end{array}$ & Sapkas et al. [41] & Low & AP and lateral X-ray & Not provided & + \\
\hline \multirow{7}{*}{$\begin{array}{l}\text { Apical vertebral rotation } \\
\quad(N=354)\end{array}$} & Pritchett et al. [8] & Low & Nash and Moe's Grade I or II & Not provided & o \\
\hline & Sapkas et al. [41] & Low & Nash and Moe's Grade I or II & Not provided & o \\
\hline & Pritchett et al. [8] & Low & Nash and Moe's Grade II or III & Not provided & + \\
\hline & Sapkas et al. [41] & Low & Nash and Moe's Grade II or III & Not provided & + \\
\hline & Kohno et al. [39] & High & Nash and Moe's Grade I-III & $r=0.502$ & + \\
\hline & Watanuki et al. [43] & Low & Perdriolle and Vidal: $5.2^{\circ}\left( \pm 3.3^{\circ}\right)$ of L3 & $p<0.05$ & + \\
\hline & Korovessis et al. [36] & High & $\begin{array}{l}\text { Perdriolle and Vidal: } 15.68^{\circ}\left( \pm 7.57^{\circ}\right) \text { of } \\
\text { apical vertebra }\end{array}$ & $\begin{array}{l}r=0.74- \\
0.83\end{array}$ & o \\
\hline \multirow{3}{*}{$\begin{array}{l}\text { Intercrest line through } \mathrm{L} 4 \\
\quad(N=230)\end{array}$} & Pritchett et al. [8] & Low & Standing AP and L X-ray & Not provided & o \\
\hline & Sapkas et al. [41] & Low & Standing AP and L X-ray & Not provided & o \\
\hline & Seo et al. [40] & High & Standing AP and L X-ray & $p<0.05$ & o \\
\hline \multirow{5}{*}{$\begin{array}{l}\text { Intercrest line through } \\
\text { L5 }(N=572)\end{array}$} & Pritchett et al. [8] & Low & Standing AP and L X-ray & Not provided & + \\
\hline & Sapkas et al. [41] & Low & Standing AP and L X-ray & Not provided & + \\
\hline & Seo et al. [40] & High & Standing AP and L X-ray & $p<0.05$ & + \\
\hline & Chin et al. [38] & High & Standing AP and L X-ray & Not provided & + \\
\hline & $\mathrm{Xu}$ et al. [18] & Low & Standing AP and L X-ray & $p<0.05$ & + \\
\hline Apex L2-3 & Sapkas et al. [41] & Low & Standing AP X-ray & Not provided & + \\
\hline Number of vertebral fractures & Kobayashi et al. [13] & High & Morphometrically $^{\mathrm{D}}$ & Not provided & o \\
\hline \multirow[t]{2}{*}{ Spondylolisthesis $(N=189)$} & Sapkas et al. [41] & Low & $\begin{array}{l}\text { Amount of displacement of the upper } \\
\text { vertebra divided by the entire width of the } \\
\text { vertebra }\end{array}$ & Not provided & o \\
\hline & Seo et al. [40] & High & $\begin{array}{l}\text { Degenerative listhesis anteriorly, posteriorly } \\
\text { or both (sagittal) }\end{array}$ & $p>0.05$ & o \\
\hline Apex translation $(N=318)$ & Xu et al. [18] & Low & $\begin{array}{l}\text { The distance from the center of the sacral } \\
\text { line to the apical vertebra (mean } 22 \mathrm{~mm} \text { ) }\end{array}$ & $p>0.05$ & o \\
\hline \multirow{4}{*}{$\begin{array}{l}\text { Lateral vertebral } \\
\text { translation } \geq 6 \mathrm{~mm}(N=183) \\
\text { or Lateral listhesis of } \mathrm{L} 3 \text { or } \mathrm{L} 4 \\
\text { (apex) }\end{array}$} & Pritchett et al. [8] & Low & $\mathrm{AP}$ and lateral view & Not provided & + \\
\hline & Korovessis et al. [36] & High & $\begin{array}{l}\text { A perpendicular tangential line is drawn on } \\
\text { the lateral edge of the apical vertebra on to } \\
\text { the line passed on the lower endplate of the } \\
\text { vertebra (\% lateral spondylolisthesis) }\end{array}$ & $\begin{array}{l}r=0.71- \\
0.75\end{array}$ & + \\
\hline & Chin et al. [38] & High & & Not provided & + \\
\hline & Seo et al. [40] & High & $\begin{array}{l}\text { The distance from the reference line of } \\
\text { laterally translated vertebral body to that } \\
\text { of the lower vertebra (lateral listhesis) }\end{array}$ & $p<0.05$ & + \\
\hline \multirow{3}{*}{$\begin{array}{l}\text { Lateral vertebral } \\
\quad \text { translation }<6 \mathrm{~mm}(N=230)\end{array}$} & Pritchett et al. [8] & Low & $\mathrm{AP}$ and lateral view & Not provided & o \\
\hline & Sapkas et al. [41] & Low & Standing, using Vernier calipers ${ }^{\mathrm{A}}$ & Not provided & o \\
\hline & Seo et al. [40] & High & $\begin{array}{l}\text { Distances from the reference line of laterally } \\
\text { translated vertebral body to that of the } \\
\text { lower vertebra }\end{array}$ & $p>0.05$ & o \\
\hline \multirow{2}{*}{$\begin{array}{l}\text { Lateral vertebral } \\
\text { translation } \geq 3 \mathrm{~mm}(=42)\end{array}$} & Kohno et al. [39] & High & Not provided & $r=0.054$ & o \\
\hline & Watanuki et al. [43] & Low & $\begin{array}{l}\text { The horizontal distance between two vertical } \\
\text { lines which were drawn from the waist to } \\
\text { the adjacent vertebra (lateral } \\
\text { spondylolisthesis) }\end{array}$ & $p<0.05$ & + \\
\hline \multirow[t]{2}{*}{ Harrington factor $(N=118)$} & Korovessis et al. [36] & High & Cobb angle divided by number of vertebrae & $r=0.96-0.99$ & + \\
\hline & Kohno et al. [39] & High & & $p>0.05$ & o \\
\hline \multirow{2}{*}{$\begin{array}{l}\text { High Cobb angle }\left(\geq 30^{\circ}\right) \\
\quad(N=203)\end{array}$} & Pritchett et al. [8] & Low & $\geq 30^{\circ}\left(<30^{\circ}\right.$ vs $\left.\geq 30^{\circ}\right)$ & Not provided & + \\
\hline & Sapkas et al. [41] & Low & $\geq 30^{\circ}\left(<30^{\circ}\right.$ vs $\left.\geq 30^{\circ}\right)$ & Not provided & + \\
\hline
\end{tabular}


Table 3 continued

\begin{tabular}{|c|c|c|c|c|c|}
\hline Determinant ( $N$ patients) & References & $\begin{array}{l}\text { Study } \\
\text { quality }\end{array}$ & Measurement methods & $\begin{array}{l}\text { Statistical } \\
\text { analysis }\end{array}$ & $\begin{array}{l}\text { Association } \\
\text { with curve } \\
\text { progression }\end{array}$ \\
\hline \multirow{6}{*}{$\begin{array}{l}\text { Low Cobb angle }\left(<30^{\circ}\right) \\
\qquad(N=314)\end{array}$} & Pritchett et al. [8] & Low & $<30^{\circ}$ vs $\geq 30^{\circ}$ & Not provided & o \\
\hline & Sapkas et al. [41] & Low & $<30^{\circ}$ vs $\geq 30^{\circ}$ & Not provided & o \\
\hline & Kohno et al. [39] & High & $>5^{\circ}-<20^{\circ}$ & $p>0.05$ & o \\
\hline & Seo et al. [40] & High & $<30^{\circ}$ & $p>0.05$ & o \\
\hline & Chin et al. [38] & High & $3^{\circ}-15^{\circ}$ vs $15^{\circ}-30^{\circ}$ & Not provided & o \\
\hline & Watanuki et al. [43] & Low & $10^{\circ}-26^{\circ}$ & Not provided & o \\
\hline
\end{tabular}

o, no correlation/no relationship found between prognostic factor and curve progression; + , positive association between prognostic factor and increased curve progression; $\rho$, Spearman correlation; $r$, Pearson's correlation; AP, anteroposterior; L, lateral; CSA, central spinal axis; JOA, Japanse Orthopedic Association; DEXA, dual energy X-ray absorptiometry

A Position of the slip vertebrae of the CSA was expressed as the ratio of the distance in $\mathrm{mm}$ from the midpoint of the slip vertebra to the CSA divided by the slip diameter in $\mathrm{mm}$

B The length of lateral osteophytes was the sum of the distances measured from the tip of the osteophyte on the upper and lower endplate to the reference line

C More than $25 \%$ decrease in anterior vertebral height compared with posterior height, more than $20 \%$ decrease in middle vertebral height compared with anterior or posterior height, or more than $20 \%$ decrease in any vertebral height compared with adjacent vertebrae

D Grade 0: no modification in vertebral trabecular pattern. Grade 1: decreased transverse trabeculae. Grade 2: decreased vertical trabeculae or unclear vertical and transverse trabeculae

to the understanding of the "depth of L5 seating" and its relationship to pelvic retroversion and curve progression.

Chin et al. observed an approximately two times higher progression rate in patients with $\mathrm{LVT} \geq 6 \mathrm{~mm}$ compared with LVT $<6 \mathrm{~mm}$ [38]. Consistent results on curve progression were found in all studies when the apical vertebra showed LVT $\geq 6 \mathrm{~mm}$ compared with $<6 \mathrm{~mm}[8,36,40]$. Interestingly, contradicting results were found by Watanuki et al. when including patients with LVT $\geq 3 \mathrm{~mm}$ [43]. A possible explanation for this might be the difference in cutoff point used. Note that LVT is a two-dimensional representation of a triaxial deformity consisting of axial rotation and lateral translation toward the convexity of the curve, which occurs predominantly at the L3-4 level [4446]. Furthermore, it has been hypothesised that vertebral rotation or "rotational instability" may be the initial trigger of DNDLS, and therefore, there might be a causal relationship. Vertebral rotation in combination with lateral spondylolisthesis (lateral vertebral translation) [36, 43, 46] or independently $[8,39,41]$ is moderately associated with curve progression (Fig. 2). Only one paper (Korovessis et al.) did not find this relationship, but their deviation from the common grading scale (i.e., the method by Nash and Moe) and relatively short follow-up time (mean 3.7 years; range 2-5 years) might account for this discrepancy [36].

\section{Insufficient evidence}

Limited, inconclusive, and conflicting evidence was found for multiple candidate prognostic factors (Fig. 2). The lack of consistent evidence for these potential prognostic factors, however, should not be interpreted as that these are not associated with curve progression.

Only two low-quality studies were available, and both found a positive correlation between curve magnitude (Cobb angle of $\geq 30^{\circ}$ ) and curve progression [8, 41]. A strong relationship between a Cobb angle of $\geq 30^{\circ}$ and curve progression has already been established in other types of scoliosis. One paper included a 40-year follow-up of AIS patients with a Cobb angle of $\geq 30^{\circ}$, and showed curve severity to be an important risk factor for curve progression in skeletally mature patients [28]. Similar results are found for idiopathic juvenile scoliosis [47]. When combining this knowledge with the data reported in the two studies on DNDLS, we speculate that a de novo degenerative lumbar scoliosis with a Cobb angle of $\geq 30^{\circ}$ will most likely progress. Conversely, in milder curves (Cobb angle $<30^{\circ}$ ), there is strong evidence (six studies) that there is no relationship with curve progression [8, 38-41, 43]. However, these findings ought not to be confused with the assumption that low magnitude DNDLS curves of $<30^{\circ}$ do not progress at all. By definition, all de novo curves started with a $0^{\circ}$ curve, and must have progressed to some degree, even if below $30^{\circ}$. This is in line with Chin et al. and Murata et al. who both demonstrated progression in DNDLS with mild curves $<30^{\circ}$, but they did not study the strength of association with curve progression [37, 38].

Although degenerative spondylolisthesis is a common condition in the population of DNDLS, only two studies have investigated the presence of degenerative spondylolisthesis and the specific role that it plays in DNDLS curve progression $[40,41]$. It remains unclear whether this 


\begin{tabular}{|c|c|c|}
\hline & $\begin{array}{l}\text { Associated with } \\
\text { DNDLS curve } \\
\text { progression }\end{array}$ & $\begin{array}{l}\text { Non-associated with } \\
\text { DNDLS curve } \\
\text { progression }\end{array}$ \\
\hline 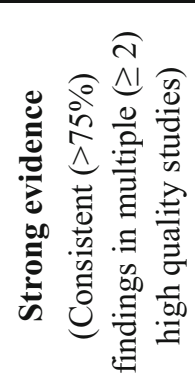 & $\begin{array}{l}\text { - Increased } \\
\text { Intervertebral disc } \\
\text { degeneration } \\
\text { - Intercrest line } \\
\text { through L5 (rather } \\
\text { than L4 or higher) } \\
\text { - Apical LVT } \geq \\
6 \mathrm{~mm}\end{array}$ & $\begin{array}{l}\text { - Osteoporosis } \\
\text { - Low Cobb angle } \\
<30^{\circ} \\
\text { - Lumbar lordosis } \\
\text { - Lateral osteophytes } \\
\text { difference } \geq 5 \mathrm{~mm}\end{array}$ \\
\hline 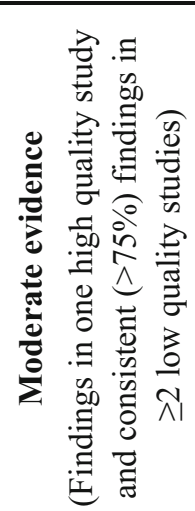 & $\begin{array}{l}\text { - AVR grade II/III } \\
\text { (Nash and Moe's) }\end{array}$ & $\begin{array}{l}\text { - Intercrest line } \\
\text { through L4 } \\
\text { - Apical LVT <6mm } \\
\text { - Direction of scoliosis } \\
\text { - Female gender } \\
\text { - Age }\end{array}$ \\
\hline \multicolumn{3}{|c|}{ AVR: Apical vertebral rotation } \\
\hline
\end{tabular}

\begin{tabular}{|c|c|}
\hline & $\begin{array}{l}\text { Insufficient evidence for } \\
\text { association with DNDLS curve } \\
\text { progression }\end{array}$ \\
\hline 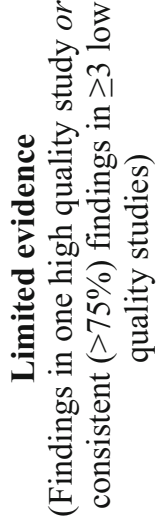 & $\begin{array}{l}\text { - BMI } \\
\text { - Sagittal balance } \\
\text { - Vertebral wedging (index) of } \\
\text { the L4 vertebra } \\
\text { - Degenerative spondylolisthesis } \\
\text { - Follow-up duration } \\
\text { - Pelvic tilt } \\
\text { - Decreased sacral inclination } \\
\text { - Thoracic kyphosis } \\
\text { - Bone atrophy } \\
\text { - Number of vertebral fractures }\end{array}$ \\
\hline 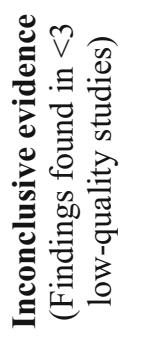 & $\begin{array}{l}\text { - AVR grade I/II (Nash and } \\
\text { Moe's) } \\
\text { - High Cobb angle } \geq 30^{\circ} \\
\text { - Secondary compensatory curve } \\
\text { at L4-S1 }\end{array}$ \\
\hline 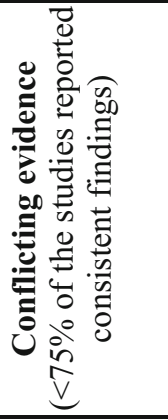 & $\begin{array}{l}\text { - Wedging of vertebra } \\
\text { - AVR (Perdriolle and Vidal) } \\
\text { - Apical LVT } \geq 3 \mathrm{~mm} \\
\text { - Apex translation } \\
\text { - Harrington factor (Cobb angle } \\
\text { devided by number of vertebrae } \\
\text { in the curve) } \\
\text { - Apex at L2-L3 } \\
\text { - Back pain/disability }\end{array}$ \\
\hline
\end{tabular}

Fig. 2 Level of evidence is shown for all associated and non-associated prognostic determinants that were identified based on Furlan et al. [34]

is related to curve progression and its role has yet to be determined (Fig. 2).

Studies of adult spinal deformity (ASD) demonstrate that positive sagittal balance is poorly tolerated and correlates with suboptimal health outcomes [48, 49]. The present review has identified limited evidence regarding the association of sagittal alignment with curve progression (Fig. 2; Table 3). As described by Aebi [7], the clinical characteristics and pathophysiology of DNDLS are different compared with other forms of ASD. This has been supported by studies which found very weak correlation between health outcomes and sagittal plane deformity, when limiting ASD to DNDLS $[50,51]$. It is, therefore, most likely that a distinction between the subtypes of ASD, such as DNDLS, should be made in determining the clinical course.

\section{Osteoporosis}

Gillespy et al. and Vanderpool et al. both found osteoporosis based on plain lateral radiographs to be correlated with curve progression in DNDLS [11, 52]. More recent research found no association with curve progression when osteoporosis was measured with DEXA scans (with a smaller margin of error) [6, 18, 39, 40]. We, therefore, conclude that despite some inconsistent reports, based on the current literature and using DEXA scans for diagnosis, there is no relationship between osteoporosis and curve progression. This seems to align with other findings that de novo degenerative scoliosis is a degenerative disorder of the intervertebral disks, and not of the bones (e.g., [7, 13]). 


\section{Direction of scoliosis}

Chin et al. found the left convex scoliosis to progress faster than the right convex scoliosis [38]. However, this result differs from other studies who found no correlation between the direction of scoliosis and curve progression $[8$, 11, 40, 41]. These contradicting findings may be explained in part by the difference between the groups. Chin et al. only looked at low magnitude DNDLS of $<30^{\circ}$, while contradicting studies included all patients with DNDLS of $\geq 10^{\circ}$ [38]. It is possible, therefore, that the direction of scoliosis may be a predictive risk factor for curve progression in the early phase of DNDLS, and not in a later phase. Unfortunately, the raw data of the contradicting studies were not available.

\section{Clinical factors}

No clinical factors (e.g., cigarette smoking, BMI, age, and gender) were identified that are related to curve progression.

\section{Limitations}

The results of this review should be interpreted cautiously. These findings are based on published data of observational studies and may be subject to various biases that may account for discrepancies found between prognostic factors and curve progression [53, 54]. Relevant (and unpublished) studies hidden in unknown databases are difficult to locate and may have been missed. Therefore, the possibility of publication bias cannot be excluded. Non-English studies were excluded in the systematic search to sufficiently assess the methodological quality. In addition, the number of prospective studies reporting on prognostic factors for DNDLS curve progression is low. There was lack of reporting of effect sizes and a large variety in definitions of DNDLS and curve progression, which made pooling of data not possible and complicated the interpretation of the reported associations. It was not possible to assess the interplay between the prognostic factors and relative contribution of each of the prognostic factors to the variance in curve progression, due to the lack of statistical methods in analyzing the prognostic value of each of the factors. In addition, these results may not be directly applicable to the individual patient. We did not study the relationship of curve progression to clinical presentation.

\section{Conclusion}

This review shows strong evidence that increased intervertebral disk degeneration, an intercrest line through L5, and lateral vertebral translation $\geq 6 \mathrm{~mm}$ are associated with DNDLS curve progression. Clinical risk factors (e.g., cigarette smoking, BMI, age, and gender) and sagittal malalignment (pelvic retroversion) and their association with curve progression have yet to be determined, and we recommend further study. We recommend obtaining an international consensus on a core set of relevant prognostic factors (clinical and radiological) and how to measure these in a standardized and valid way, so that untreated patients with DNDLS can be included in registries or long-term prospective cohort studies to help improve our understanding of the pathophysiology of curve progression in DNDLS. Although previous studies have demonstrated that progression of scoliosis may be associated with back and leg pain, more research needs to be undertaken to identify how curve progression is related to clinical course. This is crucial to establish optimal follow-up strategies and timing for therapeutic interventions for this increasing group of patients, with an increasing burden on society.

\section{Compliance with ethical standards}

\section{Conflict of interest None.}

Open Access This article is distributed under the terms of the Creative Commons Attribution 4.0 International License (http://crea tivecommons.org/licenses/by/4.0/), which permits unrestricted use, distribution, and reproduction in any medium, provided you give appropriate credit to the original author(s) and the source, provide a link to the Creative Commons license, and indicate if changes were made.

\section{References}

1. Vos T, Flaxman AD, Naghavi M et al (2012) Years lived with disability (YLDs) for 1160 sequelae of 289 diseases and injuries 1990-2010: a systematic analysis for the Global Burden of Disease Study 2010. Lancet 380:2163-2196. doi:10.1016/S01406736(12)61729-2

2. Woolf AD, Pfleger B (2003) Burden of major musculoskeletal conditions. Bull World Health Organ 81:646-656

3. Hansson EK, Hansson TH (2005) The costs for persons sicklisted more than one month because of low back or neck problems. A two-year prospective study of Swedish patients. Eur Spine J 14:337-345. doi:10.1007/s00586-004-0731-3

4. Hoy D, March L, Brooks P et al (2014) The global burden of low back pain: estimates from the Global Burden of Disease 2010 study. Ann Rheum Dis 73:968-974. doi:10.1136/annrheumdis2013-204428

5. Deyo RA (1988) Measuring the functional status of patients with low back pain. Arch Phys Med Rehabil 69:1044-1053

6. Robin GC, Span Y, Steinberg R et al (1982) Scoliosis in the elderly: a follow-up study. Spine (Phila Pa 1976) 7:355-359. doi:10.1097/00007632-198207000-00005

7. Aebi M (2005) The adult scoliosis. Eur Spine J 14:925-948. doi:10.1007/s00586-005-1053-9

8. Pritchett JW, Bortel DT (1993) Degenerative symptomatic lumbar scoliosis. Spine (Phila Pa 1976) 18:700-703. doi:10.1097/ 00007632-199305000-00004

9. Benner B, Ehni G (1979) Degenerative lumbar scoliosis. Spine (Phila Pa 1976) 4:548-552 
10. Pellisé F, Vila-Casademunt A, Ferrer M et al (2015) Impact on health related quality of life of adult spinal deformity (ASD) compared with other chronic conditions. Eur Spine J 24:3-11. doi:10.1007/s00586-014-3542-1

11. Vanderpool DW, James JI, Wynne-Davies R (1969) Scoliosis in the elderly. J Bone Joint Surg Am 51:446-455

12. Shin J-H, Ha K-Y, Jung S-H, Chung Y-J (2011) Genetic predisposition in degenerative lumbar scoliosis due to the copy number variation. Spine (Phila Pa 1976) 36:1782-1793. doi:10. 1097/BRS.0b013e318221a65f

13. Kobayashi T, Atsuta Y, Takemitsu M et al (2006) A prospective study of de novo scoliosis in a community based cohort. Spine (Phila Pa 1976) 31:178-182. doi:10.1097/01.brs.0000194777.87055.1b

14. Youssef JA, Orndorff DO, Patty CA et al (2013) Current status of adult spinal deformity. Glob spine J 3:51-62. doi:10.1055/s0032-1326950

15. Han S, Zhu Y, Wu Z et al (2013) The differently expressed proteins in MSCs of degenerative scoliosis. J Orthop Sci 18:885-892. doi:10.1007/s00776-013-0444-8

16. Carter OD, Haynes SG (1987) Prevalence rates for scoliosis in US adults: results from the first National Health and Nutrition Examination Survey. Int J Epidemiol 16:537-544

17. Kebaish KM, Neubauer PR, Voros GD et al (2011) Scoliosis in adults aged forty years and older: prevalence and relationship to age, race, and gender. Spine (Phila Pa 1976) 36:731-736. doi:10. 1097/BRS.0b013e3181e9f120

18. Xu L, Sun X, Huang S et al (2013) Degenerative lumbar scoliosis in Chinese Han population: prevalence and relationship to age, gender, bone mineral density, and body mass index. Eur Spine $\mathrm{J}$ 22:1326-1331. doi:10.1007/s00586-013-2678-8

19. Schwab F, Dubey A, Gamez L et al (2005) Adult Scoliosis: Prevalence, SF-36, and Nutritional Parameters in an Elderly Volunteer Population. Spine (Phila Pa 1976) 30:1082-1085. doi:10.1097/01.brs.0000160842.43482.cd

20. National Center for Health Statistics and Health, United States: Chartbook on Trends in the Health of Americans (2005). http:// www.cdc.gov/nchs/data/hus/hus15.pdf. Accessed 31 Dec 2015

21. McCarthy I, Hostin R, O'Brien M et al (2013) Health economic analysis of adult deformity surgery. Neurosurg Clin N Am 24:293-304. doi:10.1016/j.nec.2012.12.005

22. United States Census Bureau 2014. http://www.census.gov/ topics/population/age-and-sex.html. Accessed Dec 312015

23. De Vries B, Mullender MG, Pluymakers WJ et al (2010) Spinal decompensation in degenerative lumbar scoliosis. Eur Spine $\mathrm{J}$ 19:1540-1544. doi:10.1007/s00586-010-1368-z

24. Smith JS, Shaffrey CI, Fu K-MG et al (2013) Clinical and radiographic evaluation of the adult spinal deformity patient. Neurosurg Clin N Am 24:143-156. doi:10.1016/j.nec.2012.12.009

25. Smith JS, Shaffrey CI, Berven S et al (2009) Operative versus nonoperative treatment of leg pain in adults with scoliosis: a retrospective review of a prospective multicenter database with two-year follow-up. Spine (Phila Pa 1976) 34:1693-1698. doi:10. 1097/BRS.0b013e3181ac5fcd

26. Silva FE, Lenke LG (2010) Adult degenerative scoliosis: evaluation and management. Neurosurg Focus 28:E1. doi:10.3171/ 2010.1.FOCUS09271

27. Smith JS, Shaffrey CI, Berven S et al (2009) Improvement of back pain with operative and nonoperative treatment in adults with scoliosis. Neurosurgery 65:84-86. doi:10.1227/01.NEU. 0000347005.35282.6C

28. Weinstein SL, Ponseti IV (1983) Curve progression in idiopathic scoliosis. J Bone Joint Surg Am 65:447-455. doi:10.5035/nishi seisai.42.146

29. Moher D, Liberati A, Tetzlaff J et al (2009) Preferred reporting items for systematic reviews and meta-analyses: the PRISMA statement. BMJ 339:b2535
30. Stroup DF (2000) Meta-analysis of observational studies in epidemiology. A proposal for reporting. JAMA 283:2008. doi:10. 1001/jama.283.15.2008

31. Hayden JA, Côté P, Bombardier C (2006) Evaluation of the quality of prognosis studies in systematic reviews. Ann Intern Med 144:427-437

32. Bastick AN, Runhaar J, Belo JN, Bierma-Zeinstra SMA (2015) Prognostic factors for progression of clinical osteoarthritis of the knee: a systematic review of observational studies. Arthritis Res Ther 17:152. doi:10.1186/s13075-015-0670-x

33. Lievense AM, Bierma-Zeinstra SMA, Verhagen AP et al (2002) Prognostic factors of progress of hip osteoarthritis: a systematic review. Arthritis Rheum 47:556-562. doi:10.1002/art.10660

34. Furlan AD, Pennick V, Bombardier C, van Tulder M (2009) 2009 updated method guidelines for systematic reviews in the Cochrane Back Review Group. Spine (Phila Pa 1976) 34:1929-1941. doi:10.1097/BRS.0b013e3181b1c99f

35. Viera AJ, Garrett JM (2005) Understanding interobserver agreement: the kappa statistic. Fam Med 37:360-363. doi:10. 1109/TKDE.2008.66

36. Korovessis P, Piperos G, Sidiropoulos P, Dimas A (1994) Adult idiopathic lumbar scoliosis. A formula for prediction of progression and review of the literature. Spine (Phila $\mathrm{Pa}$ 1976) 19:1926-1932. doi:10.1097/00007632-199409000-00012

37. Murata Y, Takahashi K, Hanaoka E et al (2002) Changes in scoliotic curvature and lordotic angle during the early phase of degenerative lumbar scoliosis. Spine (Phila Pa 1976) 27:2268-2273. doi:10.1097/01.BRS.0000029261.05130.1A

38. Chin KR, Furey C, Bohlman HH (2009) Risk of progression in de novo low-magnitude degenerative lumbar curves: natural history and literature review. Am J Orthop (Belle Mead NJ) 38:404-409

39. Kohno S, Ikeuchi M, Taniguchi S et al (2011) Factors predicting progression in early degenerative lumbar scoliosis. J Orthop Surg (Hong Kong) 19:141-144

40. Seo J-Y, Ha K-Y, Hwang T-H et al (2011) Risk of progression of degenerative lumbar scoliosis. J Neurosurg Spine 15:558-566. doi:10.3171/2011.6.SPINE10929

41. Sapkas G, Efstathiou P, Badekas AT et al (1996) Radiological parameters associated with the evolution of degenerative scoliosis. Bull Hosp Jt Dis 55:40-45

42. Nash CL, Moe JH (1969) A study of vertebral rotation. J Bone Joint Surg Am 51:223-229

43. Watanuki A, Yamada H, Tsutsui S et al (2012) Radiographic features and risk of curve progression of de-novo degenerative lumbar scoliosis in the elderly: a 15-year follow-up study in a community-based cohort. J Orthop Sci 17:526-531. doi:10.1007/ s00776-012-0253-5

44. Trammell TR, Schroeder RD, Reed DB (1988) Rotatory olisthesis in idiopathic scoliosis. Spine (Phila Pa 1976) 13:1378-1382

45. Freedman BA, Horton WC, Rhee JM et al (2009) Reliability analysis for manual radiographic measures of rotatory subluxation or lateral listhesis in adult scoliosis. Spine (Phila Pa 1976) 34:603-608. doi:10.1097/BRS.0b013e31819a841e

46. Bao H, Zhu F, Liu Z et al (2014) Vertebral rotatory subluxation in degenerative scoliosis: facet joint tropism is related. Spine (Phila Pa 1976) 39:S183-S189. doi:10.1097/BRS.0000000000000494

47. Charles YP, Daures J-P, de Rosa V, Diméglio A (2006) Progression risk of idiopathic juvenile scoliosis during pubertal growth. Spine (Phila Pa 1976) 31:1933-1942. doi:10.1097/01.brs. 0000229230.68870 .97

48. Glassman SD, Bridwell K, Dimar JR et al (2005) The impact of positive sagittal balance in adult spinal deformity. Spine (Phila Pa 1976) 30:2024-2029. doi:10.1097/01.brs.0000179086.30449.96

49. Protopsaltis T, Schwab F, Bronsard N et al (2014) The T1 pelvic angle, a novel radiographic measure of global sagittal deformity, 
accounts for both spinal inclination and pelvic tilt and correlates with health-related quality of life. J Bone Jt Surg Am 96:1631-1640

50. Ploumis A, Liu H, Mehbod AA et al (2009) A correlation of radiographic and functional measurements in adult degenerative scoliosis. Spine (Phila Pa 1976) 34:1581-1584. doi:10.1097/BRS. 0b013e31819c94cc

51. Ha K, Jang W, Kim Y, Park D (2015) Clinical relevance of the SRS-Schwab classification for degenerative lumbar scoliosis. Spine (Phila Pa 1976). doi:10.1097/BRS.0000000000001229
52. Gillespy TJ, Gillespy TJ, Revak C (1985) Progressive senile scoliosis: seven cases of increasing spinal curves in elderly patients. Skeletal Radiol 13:280-286

53. Easterbrook PJ, Berlin JA, Gopalan R, Matthews DR (1991) Publication bias in clinical research. Lancet 337:867-876. doi:10. 1016/0140-6736(91)90201-Y

54. Zhang Y, Niu J, Felson DT et al (2010) Methodologic challenges in studying risk factors for progression of knee osteoarthritis. Arthritis Care Res (Hoboken) 62:1527-1532. doi:10.1002/acr.20287 